\title{
SENTIMENT ANALYSIS OF TWITTER DATA FOR DEMONETIZATION IN INDIA - A TEXT MINING APPROACH
}

\author{
Kaustav Roy, Missouri University of Science \& Technology,krgd9@mst.edu \\ Disha Kohli, Missouri University of Science \& Technology,dpk3y7@mst.edu \\ Rakeshkumar Kathirvel Senthil Kumar, Missouri University of Science \& Technology, \\ rkp58@mst.edu \\ Rupaksh Sahgal, Missouri University of Science \& Technology, rsc7d@mst.edu \\ Wen-Bin Yu, Missouri University of Science \& Technology, yuwen@mst.edu
}

\begin{abstract}
In recent years, a merely boom was witnessed on analysis of opinions from social media because usually it is very difficult to obtain such a high volume of opinions through any other normal means of collecting opinion similar to surveys, polls etc. One such social media where opinions are expressed is Twitter and it plays a significant influence on any phenomenon. Hence, an accurate method for predicting sentiments could enable us to understand public view, and the impact of the event on social and economic setup can be analyzed. These have the potential to create positive or negative impact on general mindset of the society. The main focus of this paper is to analyze the sentiments expressed on Demonetization on Twitter so that public opinions and views are extracted, analyzed and used to understand the negative and positive impact of this act on the people of India.
\end{abstract}

Keywords: Twitter, Opinion Mining, Sentimental Analysis, Demonetization, SAS

\section{INTRODUCTION}

The idea of demonetization is nothing but a well known phenomenon all over the world which enables refreshments in the on-boarded economy of a particular country and pushes the financial growth up for a better future. Whilst for a country like India which is the fastest growing economy with an average rate of $7 \%$ per year over the last two decades, is also encountered with massive corruption as well as severely victimized in terms of both internal and cross-border terrorism, meanwhile to counter that the Central Govt. of India declared suppression on two highest currency notes of 500 INR and 1000 INR to be used as legally approved entities for both online and offline cash transactions effective from Nov. 82016 onwards. But the twist lies in the fact the aforementioned declaration came from the Govt. just on the eve of Nov. 82016 without any prior notice, took the entire nation by surprise. In addition to that the bureaucrats also mentioned the recollection of the said currency notes and generation of new age alternative notes of 500INR and 2000INR currencies to be available from respective nationalized banks a day after.

This demonetization procedure took a whole new turn when the official body had to circulate the new age currency notes in such large scales that could reach out to all standards of people in the society. Moreover, the circular restrains the timeline to pull the old cash out from mass market and citizens in exchange of new age currency notes to the end of the year, i.e., $31^{\text {st }}$ Dec, 2016. Initially, the aggressive move received support from renowned economists, scholars, several bankers as well as from some international commentators. On the other hand, it was heavily criticized by members of the opposition political parties, leading to debates in parliament and triggering organized protests against the proactive approach of the government in several places across India. Demonetization was supposedly implemented to combat corruption, terrorism financing and inflation. But it was often criticized as poorly designed and implemented, with scant attention paid to the laws of the market. Thus, the act had as much negative consequences as the positives.

The aim of this study is to perform text mining on Social media to examine the impact of this demonetization from public opinions which in turn, could be used by concerned authorities to smoothen the governmental process. 


\section{LITERATURE REVIEW}

Text mining is the process of deriving useful information from natural text. Also known as text analytics, this process works by identifying various patterns in the unstructured data that can be used to bring out the underlining information. Though manual text mining was introduced in mid 1980s, the modern approach of this technique, evolved from a research by Prof. Marti A. Hearst in the late 1990s. Over the time text mining techniques have improved significantly with the help of many research and cutting-edge complex techniques. (Kiritchenko et al. 2014; Lipizzi et al. 2016).

There have been prior studies in the field of analyzing social media with text mining approaches. Hari Hara Sudhan and colleagues (2012) demonstrated the collection and summarization of tweets using the SAS ${ }^{\circ}$ macro (\%GetTweet) and then conducted sentiment analysis on the fetched tweets using SAS Text Miner. They used directed search and summarization of specific text items in SAS Text Miner. By analyzing the result, 6 clusters were formed in each group of data and were used to compare the results. Implementation of stop lists in each group removed the unwanted terms in forming clusters, and descriptive terms to understand each cluster.

Younggue Bae and Hongchul Lee (2012) demonstrated how the sentiment analysis technique can be used as a valid popularity indicator or measure. The authors developed a positive-negative measure based on their two findings. First, they distinguished between the positive and negative audiences of popular users. Second, they found that the sentiments expressed in the tweets by popular users influenced the sentiment of their audience. Lastly, the positive-negative measure was used on this influence on Granger causality analysis and to find that the time-series based positive-negative sentiment change of the audience was related to the real-world sentiment landscape of popular users.

Also, Hridoy and colleagues (2015) discussed a methodology which allows utilization and interpretation of twitter data to determine public opinions. The main focus of this research was to analyze the tweets about iphone6 to note the opinions of people based on feature specific popularity analysis and male-female specific analysis. The tweets were analyzed to be mixed but there were general consistency with outside reviews and comments.

Meire and colleagues (2016) conducted a study to measure the value of information available before and after the focal post's creation time in sentiment analysis of Facebook posts by building a sentiment prediction model which included the 'before and after' information along with the traditional post variables. The results indicated, 'before and after' information increase the model's predictive performance. Their study also showed that the most important predictors include the number of uppercase letters, the number of likes and the number of negative comments. Another interesting outcome of their research is- the more the number of comments, the more likely it is to be a negative post.

Paltoglou (2016) analyzed if sentiment analysis can be successfully used for detecting significant events that occur in the world. The author explored whether sudden changes in the positivity or negativity that keywords are typically associated with can be exploited for this purpose. He showed that the number of tweets that are used for event detection is more important factor than the number of days used to extract token frequency or sentiment averages. Focusing on detecting local events he presented results that concluded that all approaches are dependent on the level of coverage that such events receive in social media.

\section{RESEARCH METHODOLOGY}

In this paper, initial sentiment of the people of India on demonetization is analyzed with twitter data captured between $8^{\text {th }}$ Nov 2016 and $13^{\text {th }}$ Nov 2016.

\section{Aim of the Study}

This study was performed using SAS Enterprise Miner, and R with the aim of achieving the following: (1) To extract Twitter data related to the demonetization of India using various hashtags. (2) To create refined clusters of descriptive terms corresponding to the various sentiments involved. (3) To analyze the sentiments on created clusters to find if the demonetization act has public support to the date till data has been collected. 


\section{Software and Programming Used}

The following software and programming languages were used for our study: (1) SAS Enterprise Miner Workstation 12.1 (2) SAS 9.3(English) (3) R Programming and (4) R Studio.

\section{Data Collection}

Data collection process started with the identification of the hashtags that were used for the tweets related to this act. While working on collecting tweets containing the identified hashtags, Twitter further provided us with suggestions of similar or related hashtags. Many hashtags related to demonetization in India were identified this way. However, the number of hashtags there were far too many to consider. To filter out the most powerful hashtags using the hashtag analysis a website (ritetag.com) was used. This analysis gave the weightage and total hashtag exposure of the various hashtags, which were useful in identifying the most informative and useful hashtags. (Hodeghatta 2013; Saif et al. 2016)

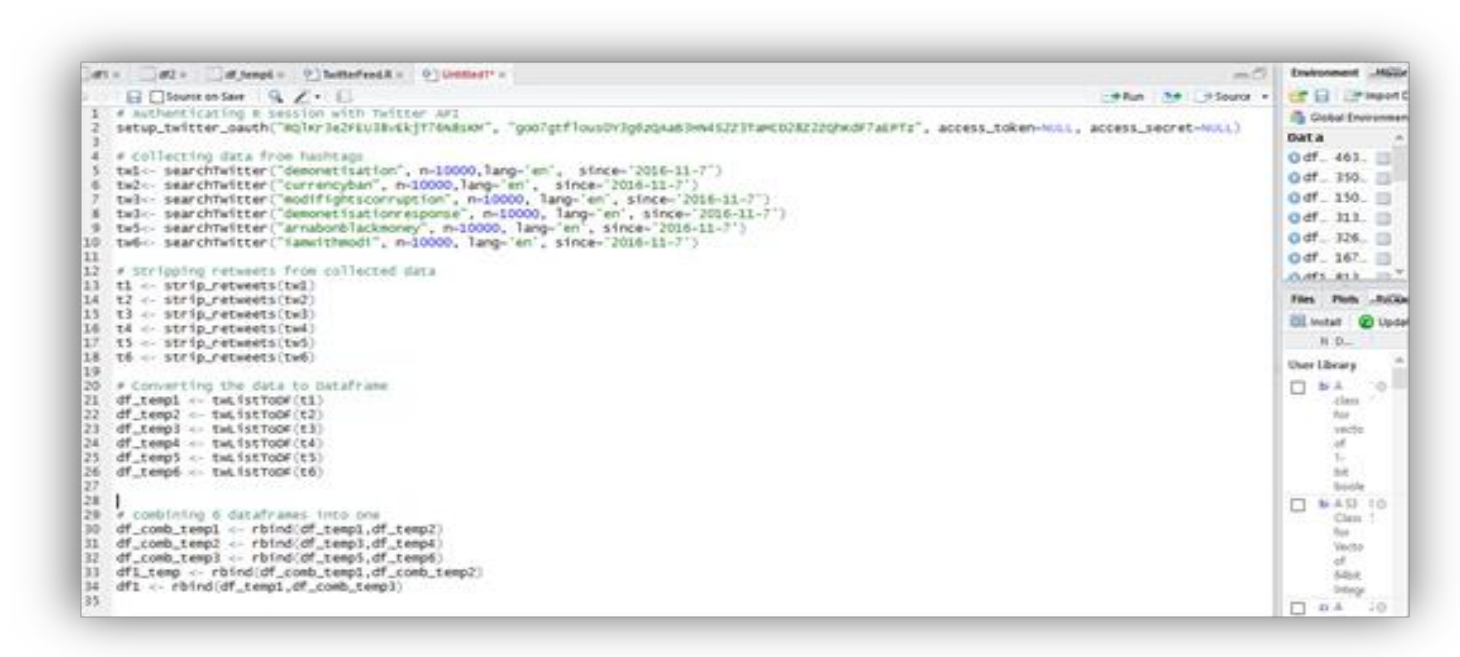

Figure 1. R code to extract Twitter data

\#Demonetization, \#Currencyban, \#Modifightscorruption, \#Demonetizationresponse, \#Arnabonbalckmoney are a few amongst the many hashtags used to identify tweets related to demonetization. Once the hashtags were confirmed we used R code (Figure 1) to extract the Twitter data and import it as a CSV file.

\section{Data Preparation}

The collected data needed to be cleansed to be used for the sentiment analysis process. Thus, $\mathrm{R}$ was used to clean up the data and keep only the necessary fields. A corpus containing all necessary fields was created and all unnecessary characters were removed leaving behind the numbers, alphabets and spaces. Later, the entire text was converted to lower case.

From a total of approximately 8000 twitter records randomly around 2000 records were picked for train data set. Those records were assigned a cluster after analyzing the sentiment manually. The assigned clusters are as follows - (1) P- Positive (2) S- Statement (3) N- Negative. The rest of the 6000 tweets (from the data-set) were placed in a different file to build a test data set.

\section{Model Preparation}

Model preparation is the process of assembling different techniques which will work as a single unit with an aim to produce an intended result. In this case, two models were prepared - one for the unsupervised method and the other for supervised method. 
Volume 18, Issue 4, pp. 9-15, 2017

For the unsupervised model (Figure 2), the first node is the data set. This node contains the data in SAS data set format which is created from the actual CSV data set.

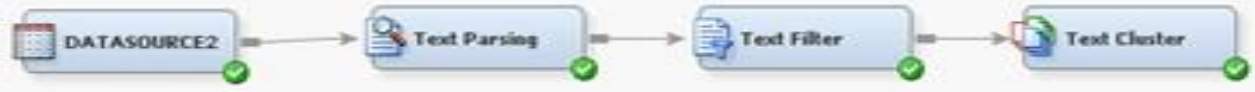

Figure 2. Model for unsupervised method

The next node is the Text Parsing node. This gives all statistical data about the terms from the data set. In addition to this, it also enables user to modify output set of parsed terms by dropping terms that are a certain parts of speech, type of entity, or attribute.

Third is the Text Filter node. It is used to filter out the duplication of terms and also the terms with similar meaning. The last node is the Test Cluster node. This is where the clustering of all the descriptive terms happens.

Next we constructed a Supervised model (Figure 3). The difference between the Unsupervised and the supervised method is that, in Supervised method we train the model with train data set containing the manually formed clusters. Once the model is trained we pass the actual test data to the model to form clusters based on its learnings from the train data set.

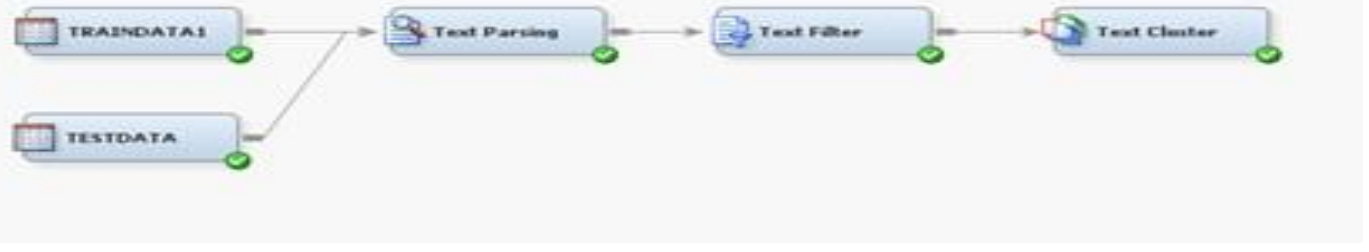

Figure 3. Model for Supervised Method

\section{Cluster Analysis}

Initially two clusters were created, positive and negative, to analyze the sentiments. Three different combinations of the settings were tried to improve the outcome and to get the best results containing the most consistent clusters. After closely analyzing the descriptive terms of the two clusters for the three different combinations, it was observed that combination 3 gave the most consistent clusters of descriptive terms with one cluster containing terms close to positive sentiment and the other containing terms close to the negative sentiment. (Table 1)

Table 1. Combinations Used for Two Cluster Unsupervised Method

\begin{tabular}{|c|c|c|c|}
\hline & Combination 1 & Combination 2 & Combination 3 (Best) \\
\hline Frequency Weight & Log & Log & Binary \\
\hline Term Weight & Entropy & Entropy & High \\
\hline SVD Resolution & Low & High & $\begin{array}{c}\text { Expectation- } \\
\text { Maximization }\end{array}$ \\
\hline Clustering Algorithm & $\begin{array}{c}\text { Expectation- } \\
\text { Maximization }\end{array}$ & $\begin{array}{c}\text { Expectation- } \\
\text { Maximization }\end{array}$ & Exact \\
\hline Exact or Minimum Number & Exact & Exact & 2 \\
\hline Number of Clusters & 2 & 2 & \\
\hline
\end{tabular}

Thus, the result of the combination 3 was selected and the records were analyzed for the correctness of the cluster number compared to the actual sentiment expressed by the text. 
On analyzing the results, it was observed that there were lots of tweets those were mere statements and didn't exhibit either of the sentiments, i.e. positive or negative. Thus, the need for another cluster that contains neutral statements arose.

Thus, the focus shifted to the three-cluster method where we grouped the neutral statements. Configuration settings of combination 3 from two clusters method was used to perform the three-cluster method analysis.

The above method gave even more consistent clusters with each exhibiting one of the three sentiments (positive, negative and neutral). On further analysis of the results it was found that the correctness of tweet-cluster mapping was up to $55 \%$. (Figure 4 )

Further the supervised method was tried to achieve even higher percentage of correctness. In this method, only the three-cluster type with three different combinations of configuration settings was performed.

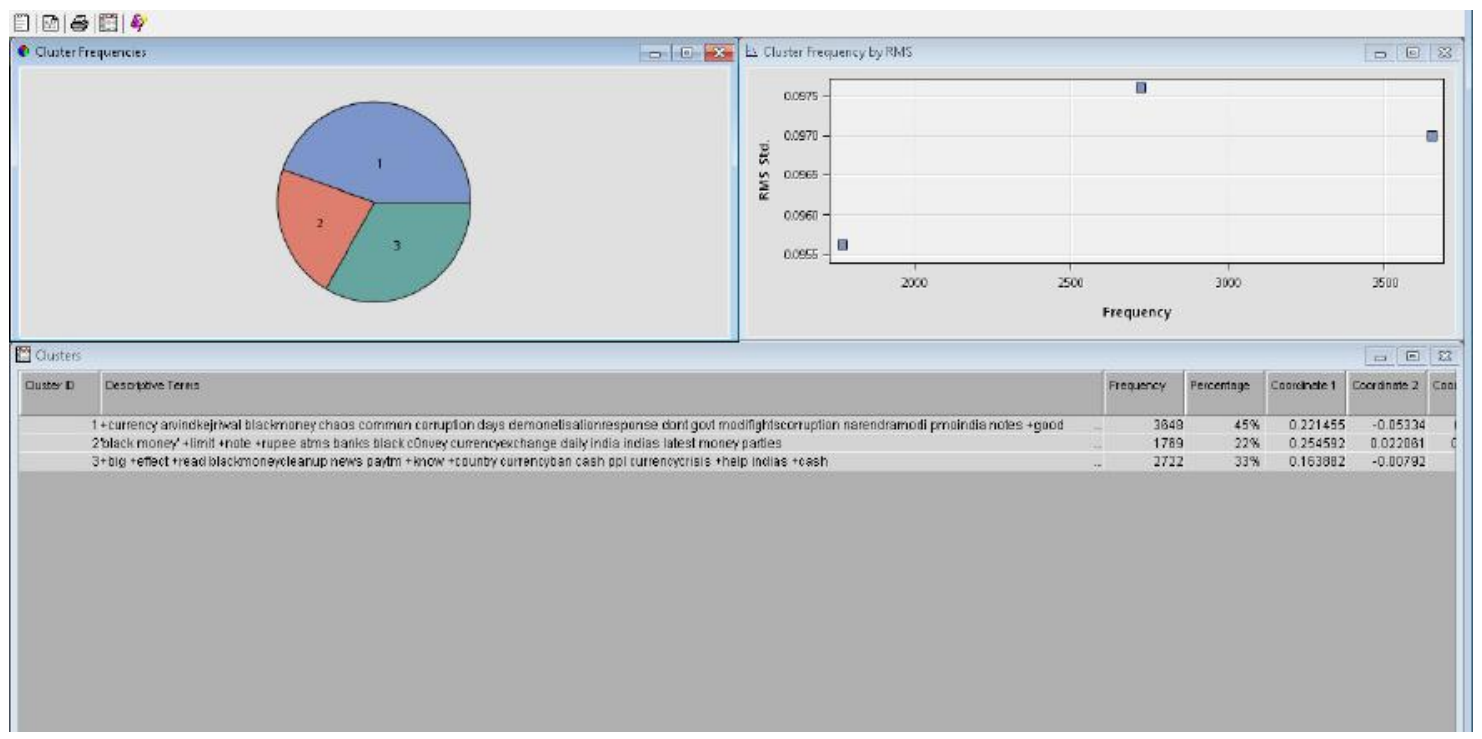

Figure 4. Descriptive Terms of Three Clusters Unsupervised Method

After closely analyzing the descriptive terms of the three clusters for the three different combinations, the second combination appeared to give the most consistent clusters of descriptive terms with each of the three clusters containing terms close to positive, neutral and negative sentiments respectively. (Table 2)

Table 2. Combinations Used for Three Cluster Supervised Method

\begin{tabular}{|c|c|c|c|}
\hline & Combination 1 & Combination 2 & Combination 3 (Best) \\
\hline Frequency Weight & Binary & Log & Binary \\
\hline Term Weight & Mutual Info. & Mutual Info. & High \\
\hline SVD Resolution & High & High & $\begin{array}{c}\text { Expectation- } \\
\text { Maximization }\end{array}$ \\
\hline Clustering Algorithm & $\begin{array}{c}\text { Expectation- } \\
\text { Maximization }\end{array}$ & $\begin{array}{c}\text { Expectation- } \\
\text { Maximization }\end{array}$ & . \\
\hline
\end{tabular}

On further analysis of the results it was found that the purity of the cluster was around $40-45 \%$, which is significantly less than unsupervised three cluster method. Thus, we decided to analyze the sentiments related to demonetization in India, using the results of unsupervised three cluster method. (Figure 5) 


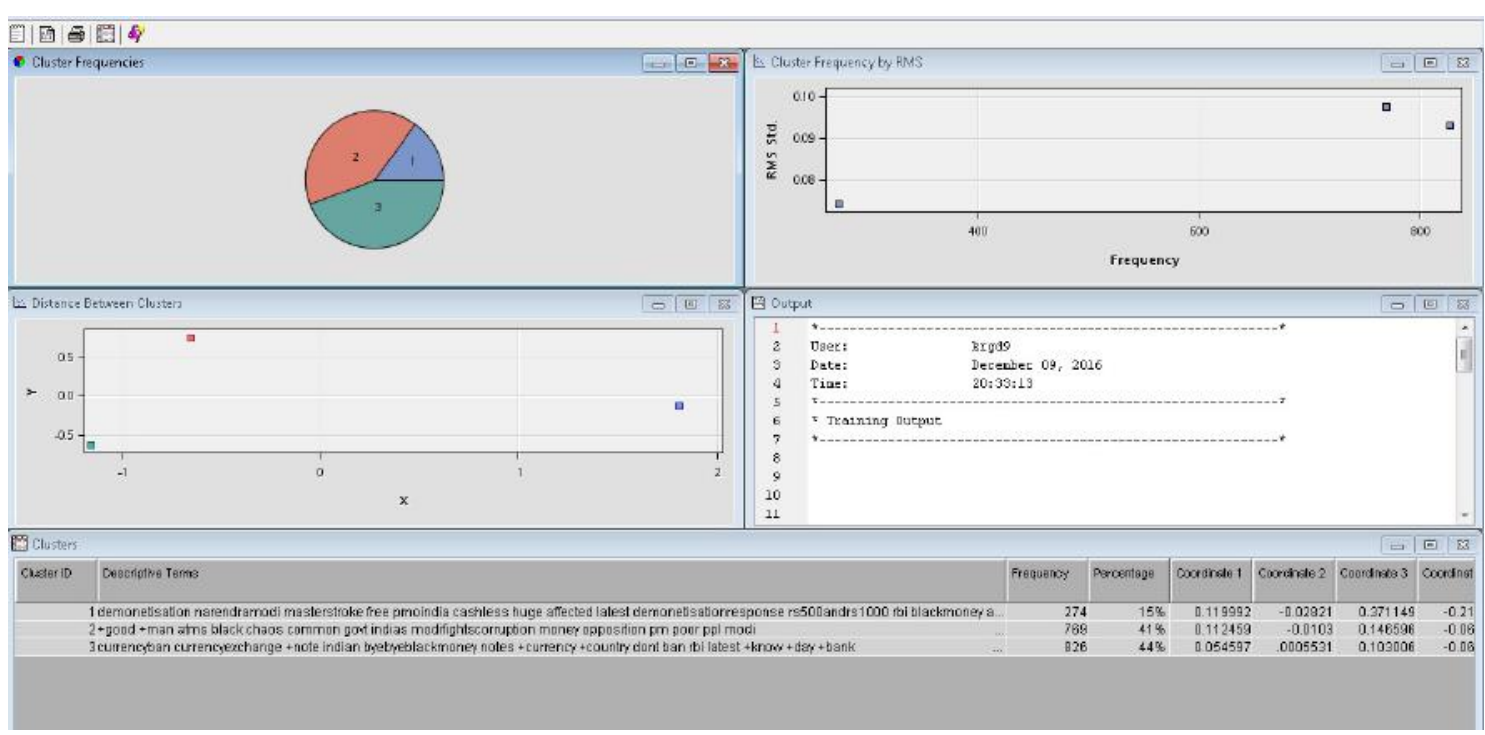

Figure 5. Descriptive Terms of Three Clusters Supervised Method

\section{RESULTS AND CONCLUSIONS}

The following results were obtained on analyzing the twitter data, in accordance to the demonetization in India (Nov 2016), for sentiments:

- Among all the tweets, approximately $45 \%$ of them were identified to be in support of this move from PM of India.

- Nearly, $22 \%$ of the tweets were identified to be neutral comments or mere statements on the effects of this act.

- With $33 \%$ of the tweets identified to be exhibiting negative sentiments most of them were identified against the execution of the act rather than the act itself.

While analyzing the sentiments of the results it was observed that there are many statements in the tweets for which the sentiments are neutral. In the remaining tweets the positive sentiment is on the higher side, approximately $50-55 \%$. The following are a couple of example of tweets that bear positive sentiment:

- "thanks \#narendramodi for \#demonetisation of course $\mathrm{v} r$ in pain expectation is 4 higher goal $\mathrm{n}$ my family 100 supports $u$ go on $\mathrm{v} r$ wid $u$ "

- "modis demonetization drive has destroyed terror networks in Kashmir \#blackmoney \#demonetisation"

Many of the tweets found to be negative were not negative to the idea of Demonetization but to the execution process. The following are a couple of examples of such tweets:

- "govts \#demonetization good idea but implementation badly planned..."

- "\#currencyban \#narendramodi situation is really bad no money in atms bad execution of such a strong good and right decision"

Sentiment analysis of social media or online texts is really important and of prime importance in many industries like online customer care service, spams filtering, contextual advertisement etc. This paper tries to capture the sentiment of the people on demonetization in India with the help of tweets. This can be helpful for the authorities to resolve the issues and make the transition smoother for people. 
Some of the future works that can be done on this paper are:

- To improve the supervised model to get better efficiency as it is a proven fact that supervised models are capable for producing results with greater precision.

- To analyze the long term impact of demonetization in India and how far this act is successful in resolving the targeted problems.

- The same process can be followed to perform sentiment analysis on GST (Goods and Service Tax) in India, which has been rolled out on 1st July 2017 and is a great leap forward from the existing tax structure.

\section{REFERENCES}

Bae, Y., \& Lee, H. (2012). Sentiment analysis of Twitter audiences: Measuring the positive or negative influence of popular twitterers. Journal of the Association for Information Science and Technology, 63(12), 2521-2535.

Hodeghatta, U. R. (2013). Sentiment analysis of Hollywood movies on Twitter. In Proceedings of the 2013 IEEE/ACM International Conference on Advances in Social Networks Analysis and Mining (pp. 14011404). ACM.

Hridoy, S. A. A., Ekram, M. T., Islam, M. S., Ahmed, F., \& Rahman, R. M. (2015). Localized twitter opinion mining using sentiment analysis. Decision Analytics, $2(1), 8$.

Kiritchenko, S., Zhu, X., \& Mohammad, S. M. (2014). Sentiment analysis of short informal texts. Journal of Artificial Intelligence Research, 50, 723-762.

Lipizzi, C., Iandoli, L., \& Marquez, J. E. R. (2016). Combining structure, content and meaning in online social networks: The analysis of public's early reaction in social media to newly launched movies. Technological Forecasting and Social Change, 109, 35-49.

Meire, M., Ballings, M., \& Van den Poel, D. (2016). The added value of auxiliary data in sentiment analysis of Facebook posts. Decision Support Systems, 89, 98-112.

Paltoglou, G. (2016). Sentiment-based event detection in Twitter. Journal of the Association for Information Science and Technology, 67(7), 1576-1587.

Saif, H., He, Y., Fernandez, M., \& Alani, H. (2016). Contextual semantics for sentiment analysis of Twitter. Information Processing \& Management, 52(1), 5-19.

Sudhan, H. H., Garla, S., \& Chakraborty, G. (2012). Analyzing sentiments in Tweets about Wal-Mart's gender discrimination lawsuit verdict using SAS ${ }^{\circledR}$ Text Miner. In SAS Global Forum, 306. Retrieved from http://support.sas.com/resources/papers/proceedings 12/306-2012.pdf 